Revista de Psicología de la PUCP. Vol. XIX, 1, 2001

\title{
Estilo de vida: avances en su medida y sus relaciones con otros conceptos psicológicos
}

\author{
María del Pilar Sánchez-López' \\ Universidad Complutense de Madrid
}

\author{
Marta Aparicio García \\ Universidad San Pablo CEU
}

\begin{abstract}
Los estudios sobre estilos de vida (EV) conducen a plantear tres investigaciones que analizan el concepto y sus relaciones con otras variables psicológicas. Primero, se presenta el análisis de las actividades realizadas por la persona, como operativización de los estilos de vida a partir del autorregistro ADIT (Autorregistro de Distribución del Tiempo) en relación con las personas con las que los sujetos realizan dichas actividades. Segundo, se plantean modificaciones del autorregistro original; por ejemplo, se eliminó el apartado que registra dónde y con quién se realiza las actividades. Tercero, se analizan las relaciones entre los EV, la satisfacción vital y las variables de personalidad en 84 personas. Estas se organizan en seis grupos, de acuerdo con las relaciones entre estos tres conceptos, como se ha venido apuntando en trabajos previos (Sánchez-López y Quiroga, 1995; Quiroga y Sánchez-López, 1997; Sánchez-López y Aparicio, 2000).

Palabras clave: estilo psicológico, estilo de vida, distribución del tiempo, satisfacción, personalidad.
\end{abstract}

Life style: progress in measuring life style and its relation with other psychological concepts Studies on life styles have driven us to consider three studies which analyse the concept and its relationship with other psychological variables. The first study presents an analysis of the activities carried out by a person, as an operational definition of life styles using ADIT self-registering (Self-Registering of Time Distribution, SRTD), considering individuals who interact with subject in those activities. In the second study, modifications to the original self-record are suggested; eg, the section that registers where and with whom are carried out the activities was eliminated. In the third study, the relationships among life styles, life satisfaction and personality variables are analysed. There were 84 persons in the sample assigned to 6 groups, depending on the relationships among these three concepts, as was previously suggested by other studies (Sánchez-López and Quiroga, 1995; Quiroga and Sánchez-López 1997; Sánchez-López and Aparicio, 2000).

Keywords: Psychological style, life style, time distribution, satisfaction, personality.

1. Doctora en psicología. Es catedrática de Psicología Diferencial y directora del Departamento de Psicología Diferencial y Psicología del Trabajo de la UCM. Es autora de 41 artículos de revistas españolas e internacionales y ha publicado 8 libros. Su línea de investigación comprende los estilos de personalidad, el bilingüismo, la cronopsicología, el rendimiento y la salud. Correo electrónico: psdif02@sis.ucm.es.

2. Licenciada en psicología por la UCM. Es profesora asociada de la UCM y de la Universidad San Pablo CEU. Asimismo, es psicóloga del colegio inglés Hastings (Madrid). Es autora de 9 artículos de revistas españolas e internacionales y ha publicado 4 capítulos de libros. Su línea de investigación comprende los estilos de personalidad y el bilingüismo. Correo electrónico: aparicio@correo.cop.es. 

El concepto estilo de vida (EV) no goza en la actualidad de una imagen integrada debido a su pluralidad de significados en el lenguaje ordinario y a la consideración del mismo como sinónimo de hábitos de comportamiento y de personalidad. El término ha sido tomado como una especie de comodín dentro del corpus del conocimiento psicológico, hecho que no impide su utilización de forma profusa. A pesar de todo, este concepto ha cobrado un nuevo auge en la psicología de la salud, en parte gracias al análisis psicológico, social y político del american way of life y a su pretensión de aislar los factores de vida nocivos para la salud y aquellos que contribuyen a la aparición o mantenimiento de determinadas enfermedades.

La introducción del término en el ámbito psicológico vino de la mano de Adler, quien adoptó el concepto de EV para reemplazar al de "plan de vida" en el intento de redefinir su noción del individuo como un actor propositivo en la vida (Rodríguez, 1992). Adler da al concepto un aspecto idiográfico, de tal forma que el concepto se aplica exclusivamente a los aspectos subjetivos del individuo (Ibáñez, 1990). El desarrollo posterior de este concepto en la psicología norteamericana de principios de siglo, centrada en el análisis conductista, utiliza preferentemente el término "hábito". Este último se diferencia del EV porque tiene una naturaleza empírica y está basado en las leyes generales del aprendizaje; tiene, asimismo, un carácter de autovalorización, además de elementalista en contraposición al concepto inconsciente y a la visión holista del EV. Sin embargo, ambos términos, EV y "hábito de vida", subrayan su principal rasgo de "ser vitales" o, lo que es lo mismo, son necesarios para la adaptación del individuo al medio (Ibáñez). Por tanto, su aplicación habitual se basa en el moderno uso comportamental, es decir, en los hábitos de consumo (Coreil, Levin y Jaco, 1992). Estos autores proponen que un conjunto de comportamientos constituye un estilo de vida si es 
compartido por un grupo social (o de estatus) y tiende a persistir a través del tiempo.

La definición quizá más convencional del EV es la de Henderson, Hall y Lipton (1980) quienes afirman que "estilo de vida de una persona es el conjunto de pautas de conducta y hábitos cotidianos de esa persona, _o más simplemente, la forma de vivir de una persona". En la actualidad, los estilos de vida son entendidos como la parte más visible y externa de los estilos (Sánchez-López, 1997). Los estilos de vida se relacionan empíricamente con otras variables psicológicas, desde las consideradas tradicionalmente diferenciales, como edad, sexo, clase social, hasta cualquier otra variable o conjunto de ellas que sirvan para caracterizar grupos de sujetos, como la satisfacción y el bienestar psicológico (Strack, Argyle y Schwarz, 1991).

El EV, en una visión más diferencialista, puede definirse como el conjunto de soluciones estratégicas que adopta el individuo para poder cumplir sus planes y objetivos globales. En la práctica adoptan la forma de patrones de vida episódicos (Shulman y Mosak, 1988; Ibañez, 1990). Shulman y Mosak definen el EV como la vía más útil para conocer a una persona, es lo que nos permite predecir su conducta, es la regla de reglas para el individuo, y es el aspecto cognitivo de la conducta. Estos autores señalan que existen temas recurrentes en la vida de cada individuo que conducen a su esencia y que se pueden rescatar agrupando algunos elementos de la conducta.

\section{La operativización del estilo de vida}

En otros trabajos anteriores se ha propuesto la distribución del tiempo como medida operativa del EV (Sánchez-López, 1997) sobre la base tanto de la racionalidad teórica (importancia del tiempo en la vida humana, McGrath, 1988), como en los datos empíricos (por ejemplo, Campbell, Converse y Rodgers, 1976; Argyle, 1987; Durán, 1987; Izquierdo, del Río y Rodríguez, 1988). Se ha construido un 
Estilo de vida: Avances en su medida y sus relaciones con otros conceptos psicológicos

instrumento de medida, el Autorregistro de Distribución de Tiempo (Sánchez-López, Quiroga, Sánchez-Herrero y Doblás, 1992³ ; Sánchez-López y Aparicio, 1997), que ha demostrado ser un instrumento útil para operativizar los estilos de vida (Sánchez-López y Aparicio, 2000).

El ADIT es un instrumento que analiza las actividades que la persona realiza a lo largo de una semana estándar de su vida (semana en la que realiza sus actividades habituales) mediante la anotación, por parte del sujeto, de sus actividades en un registro dividido en intervalos temporales de 15 minutos. Por supuesto, no se le exige que anote sus actividades en este intervalo temporal, sino que las anote cuando se produzca un cambio de actividad. El motivo de esta sugerencia es para evitar que la persona anote las actividades al final del día (como se había propuesto en los diarios de tiempo o time diary), ya que esto puede producir olvidos que falseen las verdaderas actividades realizadas puesto que la persona podría tender a anotar aquellas que considerara "mejores" o más "positivas" para ser mostradas. El ADIT es un autorregistro que requiere mucha motivación por parte de quienes lo responden, ya que-aunque después de haberlo contestado ellos se sienten satisfechos porque han podido comprobar a qué dedican el tiempo- cuando se les pide su colaboración para responder se genera cierto grado de rechazo debido a la necesidad de anotar sus actividades cada cierto tiempo.

El autorregistro está compuesto de tres áreas: la primera se refiere a las actividades que las personas realizan. Estas se han codificado con un total de treinta y seis actividades divididas en secciones tales como hogar (diecinueve tareas que comprenden a las propias domésticas, como las personales e incluido el ocio), laboral (nueve tareas), hijos (siete) y transporte (una). La segunda área registra dónde realizan estas actividades (está compuesta por dieciocho lugares) y la tercera,

3. Esta investigación fue subvencionada por el lnstituto de la Mujer (Ministerio de Asuntos Sociales, España) durante el período 1991-1993. 
con quién las realizan (siete grupos). Para facilitar su codificación e interpretación se han construido seis grupos de actividades a partir del autorregistro:

- Actividades domésticas. Se suman todas las actividades domésticas como preparar la comida, arreglar la ropa, limpiar, las compras no personales, gestión y personal doméstico.

- Actividades de ocio. Contiene la suma de lectura, ver televisión, ocio, compras personales, cenar con amigos, hablar, teléfono y cuidar plantas y animales.

- Actividades comunes. Está constituida por las actividades religiosas y las actividades sociales.

- Actividades genéricas. Comprende todas las actividades que no cumplen los requisitos de las demás variables. Son ocupaciones generales que sirven para llevar a cabo las demás funciones. Incluye los transportes, dormir, comer y arreglo personal.

- Actividades relacionadas con el trabajo. Se refiere a las distintas tareas laborales que tenían en el impreso su propio espacio y consisten en tareas mecánicas, administrativas, reuniones, de investigación, de formación de trabajo, viajes, descanso y otras tareas.

- Actividades relacionadas con los hijos. Comprende ayudar con las tareas del colegio, la comida, el aseo, ayuda en general, visitas, jugar y otras.

\section{La presente investigación}

En investigaciones previas se ha señalado la validez de este instrumento (Sánchez-López, 1997; Sánchez-López y Aparicio, 2000); en el presente trabajo, nos propusimos dar un paso más y avalar nuestra propuesta con varios estudios complementarios:

- En primer lugar, hemos analizado las actividades realizadas por los sujetos teniendo en cuenta la localización espacial (dónde 
Estilo de vida: Avances en su medida y sus relaciones con otros conceptos psicológicos

realizan las actividades) y la localización social (con quién las realizan).

- Teniendo en cuenta los resultados de estos estudios, se ha presentado un nuevo formato para el instrumento que incluye modificaciones de la primera versión del autorregistro para facilitar su aplicación.

- Con esta propuesta de modificación se presentaron los resultados del análisis de los estilos de vida, la satisfacción vital y las variables de personalidad que nos permiten concluir que el nuevo formato sigue siendo válido para medir estilos de vida; además, con él se da un nuevo paso para demostrar sus relaciones con otras variables psicológicas.

\section{Estudio 1: análisis de actividades según la localización espacial y social}

El Autorregistro, en su fase inicial (Sánchez-López et al., 1992), incluyó el análisis de las actividades realizadas por las personas; asimismo, en él se registró también con quién y dónde las hacían.

\section{Análisis de la localización social}

El primer aspecto a considerar es la localización social, es decir, con quién realizan las actividades $\operatorname{los}$ sujetos ${ }^{4}$. Esta investigación pretendía comprobar si la distribución del tiempo y "con quién" se realiza esta por parte de los sujetos estaba o no relacionada con la dedicación laboral y ocupacional de esa persona. Esto supone que puesto que el grupo de sujetos tiene diferente implicación laboral, se podrán establecer diferencias entre ellos en cuanto a su distribución del tiempo y el "con quién" lo distribuyen.

Para comprobar esta hipótesis se consideraron siete grupos posibles de opciones en la variable "con quién”: solo; con los hijos; con

4. Esta investigación se ha realizado en colaboración con Pablo Calvo, de la Universidad Complutense de Madrid. 
la pareja; con la pareja y los hijos; con amigos y compañeros; con la pareja, amigos, compañeros; y con la pareja y la familia.

En el trabajo citado se analizaron cincuenta parejas y se estudiaron meticulosamente cada uno de estos siete grupos en interacción con los seis grupos de actividades finales del ADIT comentados anteriormente (actividades genéricas, domésticas, comunitarias, con los hijos, ocio y trabajo). Como resumen de la elaboración de los perfiles para cada grupo de actividades en relación con el "con quién" exponemos en el Cuadro 1 la caracterización psicológica de cada uno de los tres grupos finales obtenidos, tanto para el tiempo laboral, como para el fin de semana.

\section{Cuadro 1}

Caracterización de los participantes según con quién realizan las actividades

\begin{tabular}{|cll|}
\hline Grupo & Laboral & Tiempo \\
\hline 1 & Domésticas solo & $\begin{array}{l}\text { Genéricas con pareja } \\
\text { Ocio solo }\end{array}$ \\
\hline 2 & $\begin{array}{l}\text { Trabajo con amigos y } \\
\text { compañeros }\end{array}$ & $\begin{array}{l}\text { Genéricas con pareja } \\
\text { Ocio con pareja e hijos }\end{array}$ \\
\hline 3 & Trabajo solo & $\begin{array}{l}\text { Genéricas con pareja } \\
\text { Ocio con pareja }\end{array}$ \\
\hline
\end{tabular}

En este Cuadro podemos observar una diferenciación clara respecto del tiempo laboral en el tipo de actividades y "con quién". Dentro de la variable tiempo laboral vemos cómo las personas que componen el grupo 1 dedican la mayor parte de su tiempo a la realización de actividades domésticas en solitario mientras que las que componen los grupos 2 y 3 se centran en la realización de actividades laborales. La diferencia entre estos dos últimos grupos radica en que en el grupo 2 esas actividades laborales son realizadas con amigos y compañeros mientras que en el grupo 3 son realizadas en solitario. Por el contrario, en el tiempo fin de semana, la única fuente de diferenciación entre los tres grupos radica en "con quién" dedican su tiempo en las actividades de ocio: el grupo 1, solo; el grupo 2 los dedica a la pareja e hijos; y el 3, exclusivamente a su pareja. 
Estilo de vida: Avances en su medida y sus relaciones con otros conceptos psicológicos

Los resultados obtenidos nos indican que el autorregistro puede ser utilizado en su versión completa o si se elimina este grupo de anotación, ya que así podemos utilizar el registro de forma más sencilla y resulta mucho más fácil de responder para los participantes.

\section{Análisis de la localización espacial}

El segundo aspecto a tener en cuenta es la localización espacial, es decir, dónde se realizan las actividades. Este aspecto se analizó como un paso previo para el análisis del uso monocrónico y policrónico del tiempo ${ }^{5}$. Al realizar el estudio de actividades monocrónicas (tiempo utilizado para realizar una tarea de manera independiente) $o$ actividades policrónicas (simultaneidad de tareas de más de $15 \mathrm{minu}$ tos; por ejemplo, ver la televisión y estudiar a la vez) se concluyó que el uso del tiempo policrónico vs el monocrónico no se puede utilizar como operativización del EV. Eso ahonda más en lo que se mencionaba anteriormente. En estos análisis se obtuvo resultados similares a los logrados en el estudio anterior, de manera que se confirma lo dicho anteriormente, es decir, podemos usar el autorregistro únicamente tomar nota de actividades o bien, si se añade la variable que contempla con quién las realizan y dónde.

\section{Estudio 2: versión multiusos del autorregistro}

La nueva propuesta de autorregistro surgió a partir de la necesidad de simplificar el viejo formato para los participantes. En tanto que las personas debían contestar sistemáticamente el cuestionario las 24 horas del día durante una semana completa, el cansancio podía llevarlas a que lo respondieran en momentos inadecuados; en la medida que ese inconveniente podía surgir, era lógico pensar que provocaría errores u olvidos a la hora de codificar.

5. Esta parte de la investigación se realizó en colaboración con Pilar Rojo del Instituto Empresa.... 
Como se ha comentado anteriormente, la localización espacial de la persona y la de quienes realizan las actividades con el sujeto que registra hacen que el registro se alargue innecesariamente. Ahora, se podrá usar esta nueva versión según los fines e intereses del investigador, de tal forma que comprobaremos si el uso exclusivo de las actividades sin estas dos áreas permitirá que los sujetos se cansen en exceso. Por este motivo, el autorregistro final estará compuesto únicamente del listado de treinta y seis actividades como lo ya lo hemos señalado. Su formato final permitirá reducir su tamaño (antiguamente el cuestionario ocupaba una DINA A3) y presentarlo en un formato de DINA A4, con ello resultaría más manejable y fácil de transportar para el participante. Para los lectores interesados en el autorregistro, pueden encontrarlo en Sánchez-López, 1997, o en Aparicio, en prensa.

\section{Estudio 3: relaciones entre los estilos de vida, la satisfacción vital y las variables de personalidad}

Una vez reducido el autorregistro, se ha realizado una investigación analizando estas tres variables (que ya han sido ampliamente estudiadas por separado, pero apenas han sido investigadas como conjunto. Los estilos psicológicos y la satisfacción vital fueron objeto de análisis conjunto en diversos trabajos (Sánchez-López y Quiroga, 1995; Quiroga y Sánchez-López, 1997; Sánchez-López y Díaz, 1998; Sánchez-López y Aparicio, 2000), mientras que el Modelo de Personalidad de Millon y, en concreto, el Inventario Millon de Estilos de Personalidad (MIPS) ya han sido analizados en otros trabajos anteriores (SánchezLópez y Aparicio, 1998; Aparicio y Sánchez-López, 1998; Aparicio y Sánchez-López, 1999; Sánchez-López y Casullo, 2000). Emmons (1991) es el único autor que ha analizado las relaciones entre estas tres variables. Cada una de ellas se han estudiado parcialmente. Las variables de personalidad se consideraban como labor novedosa.

\section{Participantes}

La muestra estuvo conformada por 84 personas. De ellas, sesenta y cuatro fueron mujeres y dieciocho hombres, todos mayores de 
19 años y menores de 59 años y con una edad media de 28.3 años. En este estudio ya no se analizaron parejas como en el que permitió validar el ADIT (Sánchez-López y Aparicio, 2000).

\section{Procedimiento}

El análisis de los estilos de vida se realizó mediante el ADIT, tal y como venimos presentándoles en este trabajo. La satisfacción vital fue medida mediante una escala likert de ítem único con valores de 0 a 10 , en donde el 0 significa muy insatisfecho y el 10 , muy satisfecho. Esta escala se presentaba para cada una de las áreas que tradicionalmente eran consideradas por los sujetos como las más significativas para valorar su satisfacción global, la familia y el trabajo. Las variables de personalidad fueron analizadas mediante el Inventario Millon de Estilos de Personalidad (MIPS); sobre él, existe la comprobación satisfactoria de su fiabilidad y validez en la bibliografía especializada (Strack, 1999; Sánchez-López y Casullo, 2000).

\section{Resultados}

El primer análisis que se ha realizado fue uno de cluster para cada uno de los grupos de variables. Se obtuvo dos grupos de personas: las satisfechos en todas las áreas y las insatisfechas, como se muestra en la Figura 1.

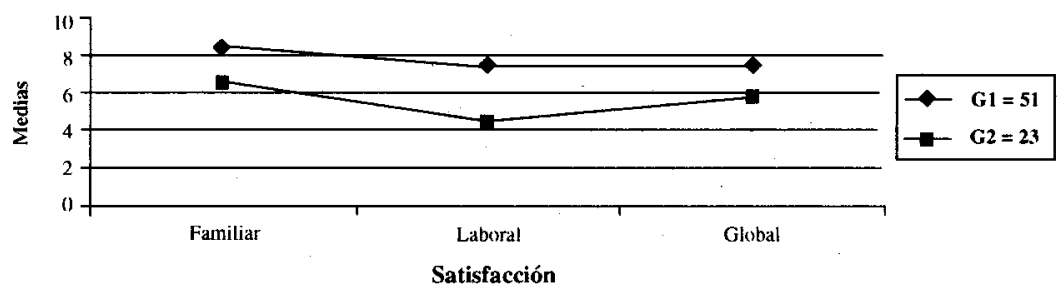

Figura 1. Análisis de cluster para satisfacción vital $(N=84)$. 
En la Figura se aprecia que aunque ambos grupos tuvieron niveles altos de satisfacción, los del segundo presentaron niveles de satisfacción más bajos que el primero en tanto se situaron en torno a una valoración de 7-8 mientras que el grupo 2 obtuvo 4-6. Para facilitar el seguimiento posterior de estos grupos se denominó al grupo 1 satisfechos y al grupo 2 insatisfechos.

En el análisis de la distribución del tiempo hemos analizado conjuntamente las actividades del tiempo laboral y las de fin de semana mediante el análisis de actividades en la variable sobre tiempo global, ya que, pese a que en estudios previos se encontró que existían perfiles diferenciales para ambos grupos (Sánchez-López y Quiroga, 1995), en ese momento nos interesaba el análisis conjunto de las actividades para conseguir grupos organizados de sujetos para realizar estudios de las tres variables investidadas: satisfacción, personalidad y estilos de vida.

En el análisis del tiempo global (TG) observamos que son significativos los grupos creados para cinco de los seis grupos de actividades. No fueron significativas las diferencias en actividades comunitarias. Se presentan estos perfiles de TG en la Figura 2.

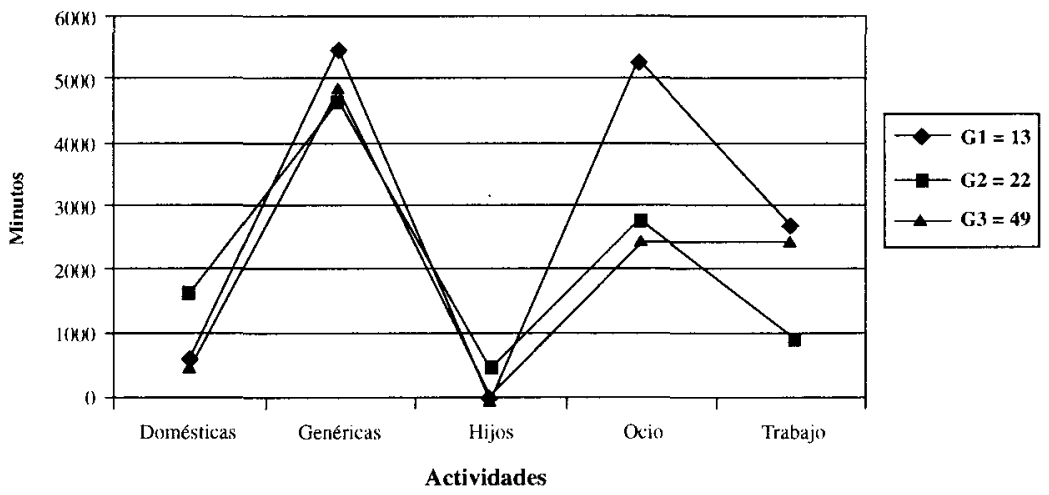

Figura 2. Análisis de cluster para estilo de vida $(N=84)$. 
Estilo de vida: Avances en su medida y sus relaciones con otros conceptos psicológicos

Se observa que el grupo 1 se compuso de trece personas y se caracterizó por dedicar más minutos que los otros a actividades genéricas, de ocio y trabajo y presentar bajos niveles en actividades de los hijos. El grupo 2 agrupó a veintidós sujetos y se caracterizó por dedicar mayor número de minutos a actividades domésticas y a los hijos, mientras que dedicó menos minutos a actividades genéricas y de trabajo. Y el grupo 3 se destacó por dedicar poco tiempo a actividades domésticas y de ocio.

Para utilizar una denominación de los grupos, el primero de distribución de tiempo se llamó "centrado-en-sí-mismo" debido a que dedicaban más tiempo a actividades propias, como el trabajo, el ocio, dormir, comer, transporte, etc. Al grupo 2 se le denominó "centradoen-los-otros", ya que dedicaban tiempo a las actividades relacionadas con los hijos y a las domésticas (eso no quiere decir que no dediquen tiempo a sí mismos). El grupo 3 fue llamado "equilibrado", ya que dedicaba una cantidad prácticamente similar de tiempo a ocio y trabajo (que es menor que en los otros dos grupos) y tiempo medio o bajo al resto de actividades.

En cuanto a las variables de personalidad, las veinticuatro variables del cuestionario MIPS fueron sometidas a análisis de cluster y se obtuvo tres perfiles de personalidad, que son diferentes significativamente en todas las escalas del MIPS, excepto en individualismo. En los gráficos 3, 4 y 5 se presentan los resultados del cluster en las tres áreas del MIPS por separado: metas motivacionales (orientación de la persona a obtener refuerzo del medio), estilos cognitivos (forma en que las personas abordan su entorno) y relaciones interpersonales (modo de comportarse con los demás), con el objeto de hacer más visibles los resultados de los análisis en cada una de las áreas.

El grupo 1 (dieciséis personas) se caracterizó por niveles medios o bajos en todas las variables de las metas motivacionales, lo que supone que no destacan respecto de los otros dos grupos en ninguna característica de esta área; en los estilos cognitivos, los participantes 
se autodescribieron utilizando sus propios pensamientos y sentimientos como recurso: son introvertidos, y niveles medios o bajos en las demás variables comparadas con los otros grupos; en las relaciones interpersonales, este grupo destacó por las altas puntuaciones en retraimiento (S8A) (falta de emotividad e indiferencia social) y vacilación (S9A) (tímidos y nerviosos en situaciones sociales). Por lo general, este grupo se situó en valores medios en todas las escalas del MIPS, pero se caracteriza por tres aspectos propios de los introvertidos, por lo que lo denominamos grupo introvertido (Figura 3).

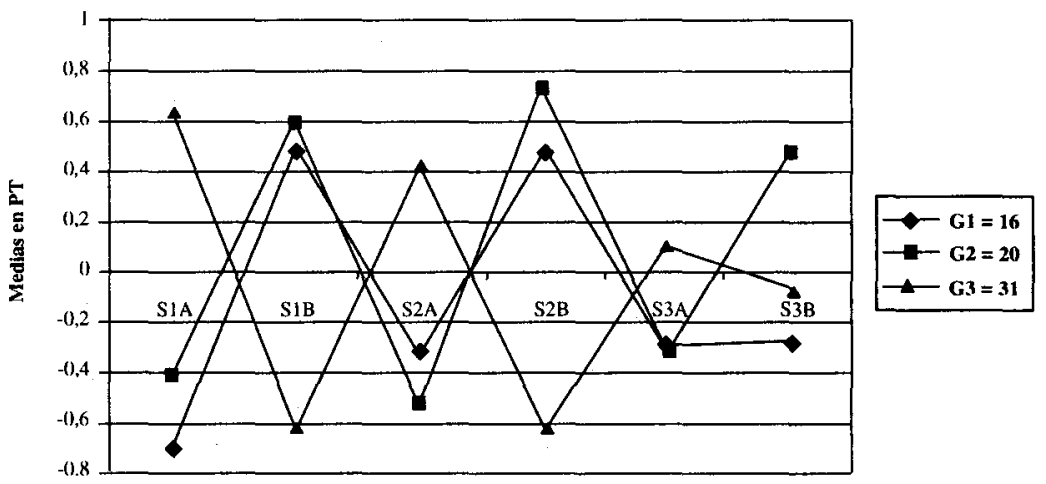

Escalas

Figura 3. Análisis de cluster para las metas motivacionales del MIPS.

SIA: E. Apertura, S1B: E. Preservación, S2A: E. Modificación, S2B: E. Acomodación, S3A: E. Individualismo, S3B: E. Protección.

El grupo 2 (veinte personas) se caracterió por altas puntuaciones en las escalas de preservación (S1B) (concentración en los problemas de la vida, agravándolos), acomodación (S2B) (no intentan dirigir sus deseos) y protección (S3B) (motivados a satisfacer primero a los demás) de las metas motivacionales; en los estilos cognitivos obtuvo altas puntuaciones en intuición (S5B) (prefieren lo simbólico y desconocido), afectividad (S6B) (forman sus juicios respecto de sus propias reacciones afectivas y guiándose por sus valores personales) e innovación (S7B) (creativos, asumen riesgos). En las relaciones 
interpersonales se caracterizaron por sus altas puntuaciones en discrepancia (S10A) (actúan de modo independiente y no conformista), en sometimiento (S11A) (habituados al sufrimiento), insatisfacción (S12A) (estados de ánimo y conductas variables) y concordancia (S12B) (simpáticos socialmente, establecen vínculos afectivos y lealtades muy fuertes). Este grupo se caracterizó por puntuar alto en los polos considerados menos adaptativos por Millon (Millon, 1994), por lo que le denominaremos desadaptativo (Figura 4).

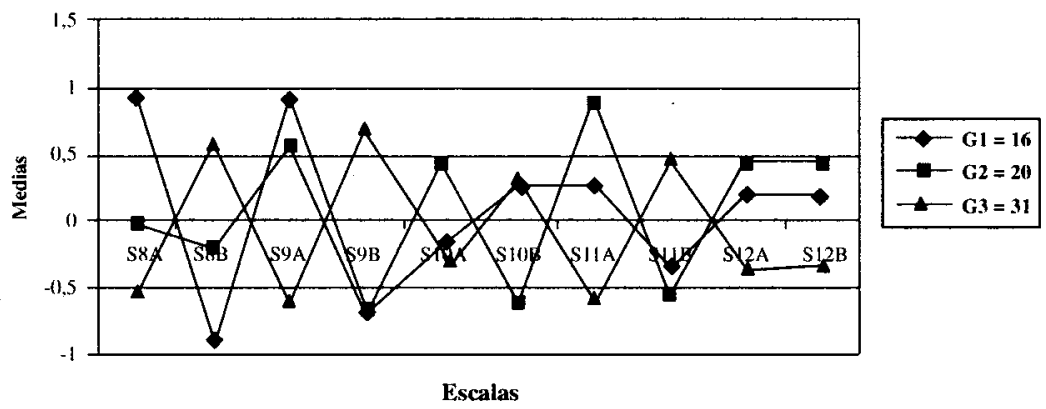

Figura 4. Análisis de cluster para los estilos cognitivos del MIPS.

S8A: E. Retraimiento, S8B: E. Comunicatividad, S9A: E. Vacilación, S9B: E. Firmeza, S10A: E. Discrepancia, S10B: E. Conformismo, S11A: E. Sometimiento, S11B: E. Control, S12A: E. Insatisfacción, S12B: E. Concordancia.

El grupo tres (treinta y un personas) obtuvo altos niveles en apertura (S1A) (optimistas en cuanto al futuro), modificación (S2A) (el futuro depende de ellos, por eso intentan cambiar su entorno para conseguir sus deseos) e individualismo (S3A) (orientados a sus propias necesidades sin preocuparse de los demás) en las metas motivacionales; en los estilos cognitivos presentaron altas puntuaciones en extroversión (S4A) (recurren a los demás para buscar estimulación), sensación (S5A) (derivan sus conocimientos de lo concreto), reflexión (S6A) (procesan los conocimientos por medio de la lógica y lo analítico) y sistematización (S7A) (transforman información nueva adecuándola a lo conocido); $y$ en las relaciones interpersonales mostraron altas puntuaciones en comunicatividad (S8B) (buscan estimulación y 
atención), firmeza (S9B) (creen ser talentosos y competentes), conformismo (S10B) (respetan la autoridad) y control (S11B) (enérgicos, dominantes y socialmente agresivos). Este grupo puntuó más alto que los otros dos grupos en los polos más adaptativos del MIPS por lo que fue denominado adaptativo.

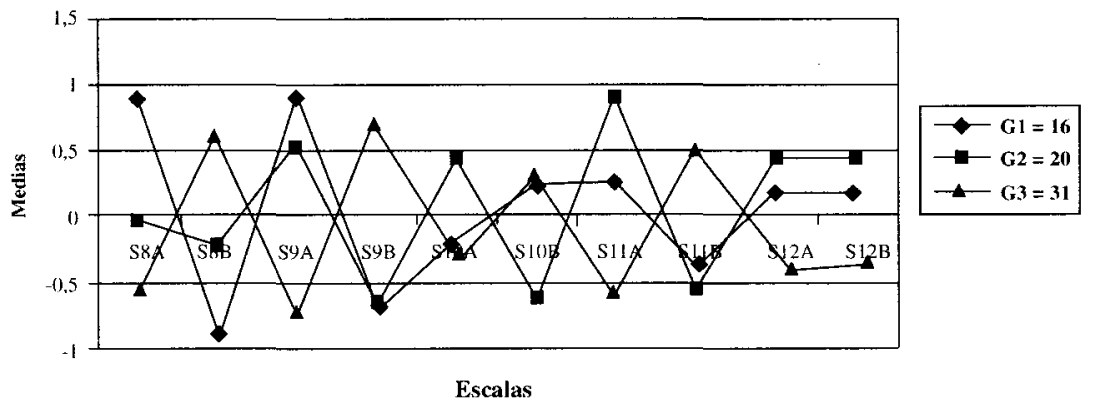

Figura 5. Análisis de cluster para las relaciones interpersonales del MIPS.

S8A: E. Retraimiento, S8B: E. Comunicatividad, S9A: E. Vacilación, S9B: E. Firmeza, S10A: E. Discrepancia, S10B: E. Conformismo, S11A: E. Sometimiento, S11B: E. Control, S12A: E. Insatisfacción, S12B: E. Concordancia.

Una vez obtenidos los grupos mediante el cluster, analizamos las relaciones entre las variables mediante tablas de contingencia. Para este análisis usamos los grupos formados al realizar los análisis de clusters, de tal forma que trabajamos con los dos grupos obtenidos en satisfacción, los tres obtenidos en el análisis del tiempo global y los tres de las puntuaciones típicas de las escalas del MIPS. En primer lugar, se relacionaron MIPS y TG para después obtener una tabla de contingencia de las tres variables conjuntamente.

En el Cuadro 2 se aprecia que los participantes pertenecen a los diferentes grupos de las dos variables. El nivel de significación de chi-cuadrado no fue significativo, aunque el coeficiente de contingencia entre las dos variables fue de 0,306. Esto supone que estos dos conceptos estuvieron poco relacionados en la población con la que trabajamos. Se obtuvo nueve grupos de personas, cinco de los cuales tenían más de cinco miembros. 
Estilo de vida: Avances en su medida y sus relaciones con otros conceptos psicológicos

\section{Cuadro 2}

Tabla de contingencia para personalidad y tiempo global

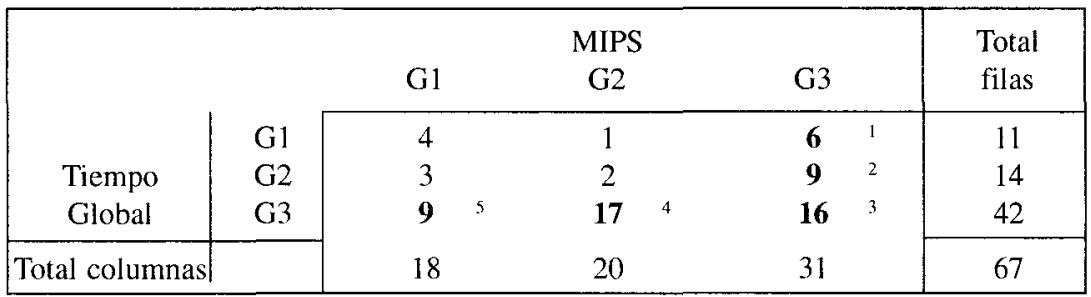

Nota. Los superíndices del 1 al 5 son los grupos significativos en la tabla de contingencia.

De estos cinco grupos, tres de ellos pertenecieron al grupo adaptativo del MIPS, es decir, aquel grupo de sujetos que puntuaban más alto en los polos más adaptativos del cuestionario. Cada uno de estos grupos perteneció a uno de los grupos de TG. Los otros dos grupos pertenecieron al grupo equilibrado de TG, esto suponía que dedicaban un tiempo medio a casi todas las actividades y tenían dos niveles de MIPS: el grupo introvertido (grupo 5) y el grupo desadaptativo (grupo 6). Así surgieron las combinaciones de grupos siguientes:

- Grupo 1: Adaptativo y Centrado-en-sí-mismo.

- Grupo 2: Adaptativo y Centrado-en-otros.

- Grupo 3: Adaptativo y Equilibrado.

- Grupo 4: Desadaptativo y Equilibrado.

- Grupo 5: Introvertido y Equilibrado.

A continuación incluimos en el análisis anterior la variable satisfacción. Para ello, hemos utilizado los cinco grupos mayoritarios de esta tabla de contingencia, ya que el resto de las casillas agrupan un número insuficiente de personas. De esta manera obtuvimos diez grupos de personas a partir del cruce de los grupos de cada variable. Sin embargo, en uno de estos grupos no se incluyó a ninguna persona y en otros tres se consideraron menos de cinco. Esto se debe a que hay muchos grupos de personas y que nuestra muestra se compone únicamente de 84 sujetos, 
con lo que en el cruce de las variables es lógico que haya casillas vacías en la tabla, ya que se van perdiendo sujetos en los diferentes análisis que se van realizando. La relación entre las tres variables fue discreta (3.99) y tuvo baja intensidad de relación (0.27). Los datos se presentan en el Cuadro 3.

\section{Cuadro 3}

Tabla de contingencia para satisfacción, tiempo global y personalidad

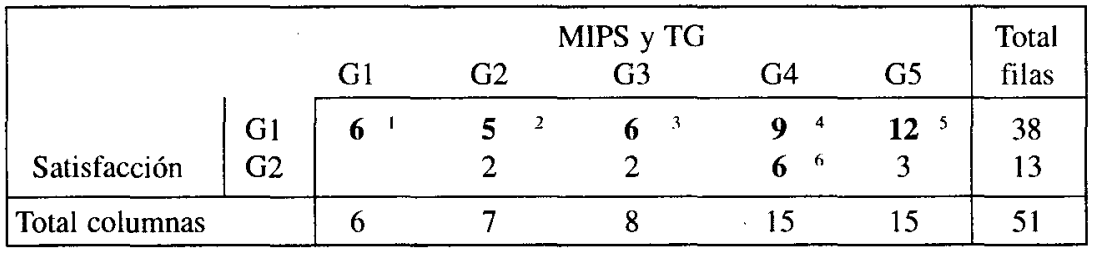

Nota. Los superíndices del 1 al 5 son los grupos significativos en la tabla de contingencia.

Los cinco grupos satisfechos. agruparon a más de cinco personas en MIPS y TG. Solo hubo un grupo insatisfecho que se caracterizó por ser desadaptativo en MIPS y equilibrado en TG. La caracterización de estos grupos fue la siguiente:

- Grupo 1: satisfechos, centrado-en-sí-mismo y adaptativo.

- Grupo 2: satisfechos, centrado-en-otros y adaptativo.

- Grupo 3: satisfechos, equilibrado e introvertidos.

- Grupo 4: satisfechos, equilibrado y desadaptativos.

- Grupo 5: satisfechos, equilibrado y adaptativos.

- Grupo 6: insatisfechos, equilibrado y desadaptativos.

Por tanto y teniendo en cuenta que existen casillas vacías y que el análisis de la significación fue discreto, se puede concluir que las personas de este grupo están satisfechas con su vida pese a que dedican su tiempo a diferentes ocupaciones, independientemente de las variables de personalidad que tengan. Solo existió un grupo de personas insatisfechas que tuvo puntuaciones en personalidad desadaptativa 
Estilo de vida: Avances en su medida y sus relaciones con otros conceptos psicológicos

y dedicaba tiempo medio a diferentes actividades sin que haya nada que destaque en su distribución del tiempo.

\section{Conclusiones}

En este trabajo se presentan nuevos aportes a los estudios anteriores sobre el autorregistro ADIT, por lo que ya se han señalado las principales conclusiones parciales que se han ido obteniendo. Una conclusión general obtenida de estas propuestas es que el autorregistro se muestra como un instrumento válido para ser usado como medida operativa del estilo de vida del sujeto, ya que nos permite analizar las actividades realizadas por los participantes y se obtienen grupos de sujetos diferentes.

Es importante señalar que autores clásicos en el estudio de las variables de personalidad como Costa y McCrae señalan que en las relaciones entre personalidad y estado de ánimo existe cierta estabilidad a lo largo de la vida de una persona de tal forma que después de 30 años una persona sigue reflejando unos rasgos de personalidad y un estado de ánimo similar al que mostró en la primera medición. Así, estos autores señalan que la "afectividad se puede predecir con años de antelación, no por el conocimiento de antemano de situaciones que produjeron respuestas afectivas, sino por la comprensión de las bases disposicionales de la afectividad: los rasgos de personalidad" (Costa y McCrae, 1998). La continuación de esta línea de trabajo permitirá confirmar estas investigaciones además de señalar que existen otros aspectos que influyen en esta relación si nos apoyamos, quizá, en que los estilos de vida interactúan con la personalidad y los estados de ánimo para elaborar un patrón de conducta de la persona que lleva al sujeto a definir su vida de forma o no satisfactoria.

A lo largo del artículo se han señalado importantes relaciones con otros conceptos que permitirán componer un modelo integrado del sujeto, puesto que, como se ha comprobado, no podemos separar 
las áreas de las personas como habitualmente se hacía en las investigaciones psicológicas. Por este motivo, nuestras líneas futuras de actuación irán encaminadas al análisis de diferentes grupos de sujetos que nos permitan determinar más claramente las relaciones entre los estilos de vida, la satisfacción vital y las variables de personalidad, así como encontrar diferentes "tipos" de sujetos según las relaciones entre estas tres áreas.

\section{Referencias}

Aparicio, M. (en prensa). ¿Cómo somos, en qué ocupamos nuestro tiempo y cuán felices nos sentimos? Aceptado para su publicación en la Revista de Psicopatología y Psicología Clínica.

Aparicio, M. y Sánchez-López, M. P. (1998). El Inventario Millon de Estilos de Personalidad: su fiabilidad y validez en España y México. Revista Mexicana de Psicología, 15 (2), 103-117.

Aparicio, M. y Sánchez-López, M. P. (1999). Los estilos de persona-

lidad: su medida a través del Inventario Millon de Estilos de Personalidad. Anales de Psicología, 15 (2), 191-211.

Argyle, M. (1987). The psychology of happiness. Londres: Methuen. Campbell, A., Converse, P. E. y Rodgers, W. L. (1976). The quality of American life: percepcions, evaluations and satisfactions. Nueva York: Bursell Sage Foundation.

Coreil, J., Levin, J. S. y Jaco, E. G. (1992). Estilo de vida. Un concepto emergente en las ciencias sociomédicas. Clínica y Salud, 3 (3), 221-231.

Durán, M. A. (1987). De puertas adentro. Madrid: Instituto de la Mujer. Emmons, R. A. (1991). Personal strivings, daily life events and psychological and physical well-being. Journal of Personality, 59 (3), 453-472.

Henderson, J. B., Hall, S. M. y Lipton, H. L.(1980). Changing selfdestructive behaviors. En G. Stone, F. Cohen y N. Adler (Eds.), Health Psychology. San Francisco: Jossey Bass. 
Estilo de vida: Avances en su medida y sus relaciones con otros conceptos psicológicos

Ibañez, E. (1990). Psicología de la salud y estilos de vida. Valencia: Promolibro.

Izquierdo, J., Del Río, O. y Rodríguez, A. (1988). La desigualdad de las mujeres en el uso del tiempo. Madrid: Ministerio de Asuntos sociales de España-Instituto de la Mujer.

Quiroga, M. A. y Sánchez-López, M. P. (1997). Análisis de la Insatisfacción familiar. Psicothema, 9 (1), 69-82.

McGrath, J. (1988). The social psychology of time. Guilford: Guilford Press.

Rapoport, R. y Rapoport, R. N. (1969). The dual career family: a varient pattern and social change. Human Relations, 22, 3-30.

Rodríguez Marín, J. (1992). Estilos de vida y salud. Clínica y Salud, 3 (3), 233-237.

Sánchez-López, M. P. (1997). El estilo psicológico como base del estudio de la diversidad humana: un ejemplo basado en los estilos de vida. Revista de Psicología, 2, 223-252.

Sánchez-López, M. P. y Aparicio, M. E. (1997). Autorregistro de distribución de tiempo: modificaciones del original. Informe de investigación interno del Departamento de Psicología Diferencial y del Trabajo, no publicado.

Sánchez-López, M. P. y Aparicio, M. E. (1998). Inventario Millon de Estilos de Personalidad: su fiabilidad y validez en España y Argentina. Revista Iberoamericana de Diagnóstico y Evaluación Psicológica, 4 (2), 87-110.

Sánchez-López, M. P. y Aparicio, M. (2000). La distribución del tiempo como medida operativa de los estilos de vida. Revista Científica del Colegio de Psicólogos de San Juan, 2 (2), 3653.

Sánchez-López, M. P. y Casullo, M. M. (2000). Los estilos de personalidad: una perspectiva iberoamericana. Buenos Aires: Miño y Dávila.

Sánchez-López, M. P. y Díaz, J. F. (1998). Relaciones entre estilo de vida y satisfacción vital. Revista de Psicología, 16 (1), 105112. 
Sánchez-López, M. P. y Quiroga, M. A. (1995). Relaciones entre satisfacción familiar y laboral: variables moduladoras. Anales de Psicología, 11 (1), 65-78.

Sánchez-López, M. P., Quiroga, M. A., Sánchez-Herrero, S. A. y Doblas, T. (1992). El reparto de responsabilidades del hogar entre los miembros de la pareja en tres tipos de familia representativos de la situación social española: parejas de doble carrera, doble ingreso y tradicionales. Prensa Académica. Investigación subvencionada por el Instituto de la Mujer.

Shulman, B. H. y Mosak, H. H. (1988). Manual for life style assessment. Muncie, IN: Acelerated Development.

Strack, S. (1999). Special Series: Millon's Evolving Personality Theory and measures. Journal of Personality Assessment, 72 (3), 323-469.

Strack, F., Argyle, M. y Schwarz, N. (1991). Subjective well-being an interdisciplinary pespective. International Series in Experimental Social Psychology, $\mathrm{n}^{\circ}$ 21. Londres: Pergamon Press. 